\title{
EFECTO DEL TAMAÑO DE PROBETA SOBRE EL CIZALLE Y LA ADHESIÓN EN UNIONES CON ACETATO DE POLYVINILO
}

\section{EFFECT OF TEST PIECES DIMENSION ON SHEAR STRENGTH AND ADHESION IN POLYVINYL ACETATE JOINTS}

\author{
Héctor Cuevas $D^{1}$, Hernán Poblete $W^{2}$.
}

\begin{abstract}
RESUMEN
El comportamiento de las uniones encoladas de tableros enlistonados se determina según los procedimientos de la Norma ASTM D-5751. Cuando el espesor de los tableros no permite obtener probetas de las dimensiones normalizadas, se debe adaptar la dimensión que no es factible lograr y realizar los ensayos sometiendo a esfuerzo un área inferior a la especificada. Para establecer el efecto del tamaño de la probeta sobre los valores de resistencia y adhesión, se realizaron ensayos en uniones de Pinus radiata, con adhesivos del tipo acetato de polivinilo (PVA), empleando tres tamaños de probetas, uno de los cuales correspondió al normalizado. El análisis estadístico indica que no hay diferencias significativas $(\mathrm{P}>0,05)$ entre los diferentes tamaños de probetas "climatizadas". Al someter las probetas a tratamientos de envejecimiento acelerado, los resultados presentan diferencias significativas $(\mathrm{P}<0,05)$.
\end{abstract}

Palabras clave: Tableros enlistonados, cizalle, adhesión, dimensión de probetas.

\begin{abstract}
For the determination of properties of block boards, ASTM D-5751 standards are applied. When board thickness does not achieve that specified by ASTM, test specimens with a lower shear surface must be prepared. In order to determinate the effect of shear surface on shear strength and adhesion, test specimens with three different shear surfaces, one of them the specified by ASTM, were tested. Boards were made up with Pinus radiata and jointed with polyvinyl acetate (PVA). Results showed that when no artificial aging was applied, no significant differences were founded. After treatments under boiling water or vacuum and pressure statistically significant differences were denoted.
\end{abstract}

Key words: Block boards, shear strength, adhesion, test specimens dimension.

\footnotetext{
${ }^{1}$ Instituto de Tecnología de Productos Forestales. Universidad Austral de Chile. Valdivia. Chile ${ }^{2}$ Instituto de Tecnología de Productos Forestales. Universidad Austral de Chile. Valdivia. Chile Autor para correspondencia: hcuevas@uach.cl Recibido: 24.05.2007.Aceptado: 23.08.2007.
} 


\section{INTRODUCCION}

Los tableros enlistonados son un elemento importante en la fabricación de muebles. En Chile son numerosas las empresas que fabrican este producto con madera de Pinus radiata, tanto para el mercado interno como para exportación utilizando adhesivos tipo PVA.

En el control de calidad de este producto se considera, fundamentalmente, el aspecto del tablero y el comportamiento mecánico de las uniones. Para la determinación de la calidad de las uniones se trabaja principalmente con la norma ASTM D-5751 (American Society for Testing and Materials, 1995) o las normas EN 204 y EN 205 (Comité Europeo de Normalización, 1993).

Ambas normas tienen ventajas y desventajas. La norma ASTM es muchas veces preferida por establecer como meta de resistencia un porcentaje de la resistencia de la madera maciza, sin unión (Poblete y Cuevas, 1998).

La norma ASTM establece procedimientos de laboratorio para determinar la resistencia de las uniones ante esfuerzo de cizalle en el plano de encolado. Se complementa con la medición de la adhesión, es decir, el porcentaje de desgarro de fibras o falla de la madera en el plano de encolado sometido a esfuerzo.

De acuerdo a las indicaciones de la norma, las probetas deben ser climatizadas previo al ensayo y considerar tratamientos que simulan un envejecimiento acelerado.

La geometría y dimensiones de la probeta establecidas en la Norma ASTM D-5751 se muestran en la figura 1 .

Para obtener probetas de un tablero con las dimensiones normalizadas se requiere de un espesor de tablero mayor o igual a 50,8 $\mathrm{mm}$. Comercialmente se producen hasta $22 \mathrm{~mm}$. Lo anterior significa que normalmente se obtienen muestras que no cumplen, en el plano sometido a esfuerzo, con una de las dimensiones normalizadas.
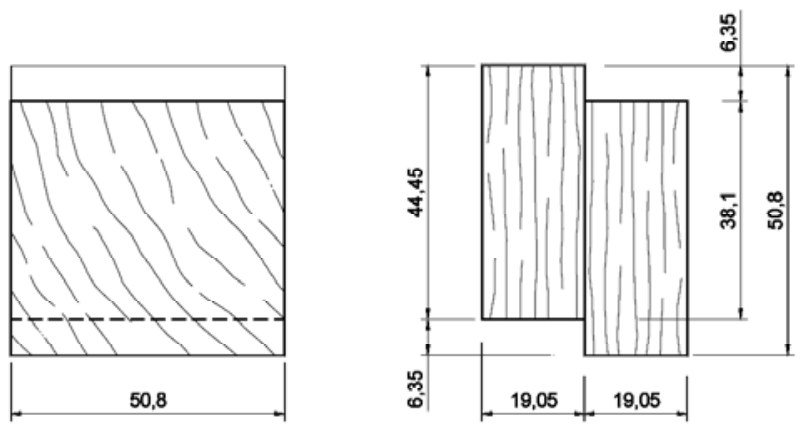

Figura 1: Forma y dimensiones de la probeta segœn norma ASTM D-5751.

Considerando esta situación se desarrolló el presente trabajo, teniendo como objetivo principal determinar si el tamaño de la probeta tiene un efecto sobre los valores unitarios de resistencia y adhesión de las uniones encoladas bajo las condiciones de ensayo normalizadas. 


\section{MATERIAL Y METODO}

Se contó con doce tableros enlistonados de $55 \mathrm{~mm}$ de espesor confeccionados con madera de Pinus radiata y cuatro formulaciones comerciales de adhesivos tipo PVA para uso húmedo (tres tableros con cada adhesivo). Los adhesivos se identificaron con las letras: A, B, C, y D.

De cada uno de los tableros se obtuvo 20 muestras. Con cada una de éstas se prepararon probetas respetando el alto de la sección indicada por la norma ASTM D-5751 y variando el ancho, para obtener así distintas áreas sometidas a esfuerzo de cizalle. Como se muestra en la figura 2, los anchos de probeta fueron 50,8 $\mathrm{mm}$ (probeta normalizada), 30,0 $\mathrm{mm}$, y 18,0 $\mathrm{mm}$.

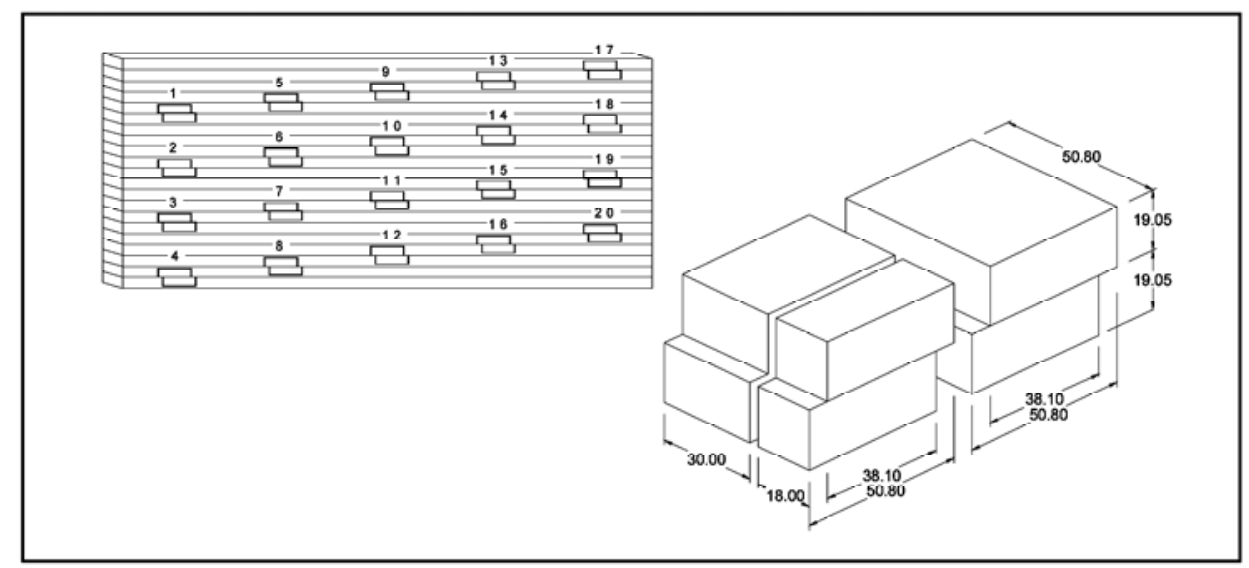

Figura 2: Lugares de obtención de las muestras y dimensiones de las probetas ensayadas.

Al variar el ancho de las probetas se obtuvieron superficies sometidas al esfuerzo de cizalle de: 19,$35 ; 11,43$ y $6,86 \mathrm{~cm}^{2}$ respectivamente.

Considerando que se trata de adhesivos para uso húmedo, todas las probetas fueron climatizadas hasta peso constante en ambiente con $20 \pm 2{ }^{\circ} \mathrm{C}$ de temperatura y $65 \pm 5 \%$ de humedad relativa del aire. Posteriormente fueron separadas en tercios, dos de los cuales fueron sometidos, por separado, a los siguientes tratamientos:

Ebullición: Inmersión en agua hirviendo durante 4 horas, secado en estufa a $63 \pm 3{ }^{\circ} \mathrm{C}$ con circulación de aire durante 2 horas, segunda inmersión en agua hirviendo por 4 horas, seguida de un enfriado en agua corriente a $18-27^{\circ} \mathrm{C}$ por un lapso de 1 hora. Ensayo antes de 60 minutos.

Vacío - Presión: 30 minutos bajo agua a $18-27^{\circ} \mathrm{C}$ con un vacío de $84.4 \mathrm{KPa}$ y 30 minutos bajo agua con una presión de $75 \pm 2$ psi. Ensayo de inmediato en condición húmeda.

Posterior al climatizado y los tratamientos, se determinó resistencia y desgarro de fibras en las 720 probetas. El ensayo se realizó en una máquina universal de ensayos mecánicos utilizando el dispositivo especialmente diseñado para este efecto, descrito en la norma ASTM D-5751. Este accesorio consiste en una cizalla con asiento autoalineador para asegurar una distribución lateral uniforme.

La carga se aplicó, de acuerdo con la norma, en movimiento continuo y uniforme a una velocidad de 0,5 pulgadas $(12,8 \mathrm{~mm}) / \mathrm{min} . \pm 10 \%$. 
La adhesión se evaluó, según el procedimiento normalizado, determinando el área en la cual la falla se produce en la madera y relacionando ésta porcentualmente con el área total. Esta situación se conoce como porcentaje de desgarro de fibras.

Los valores de resistencia y los porcentajes de desgarro de fibras obtenidos fueron procesados, en forma separada, por el paquete estadístico StatGraphics Plus. Mediante este software se realizó un análisis de varianza (ANOVA) para determinar la existencia o no de diferencias significativas entre los parámetros determinados y las áreas sometidas a esfuerzo para los tratamientos considerados.

De existir diferencias, para definirlas, se realizaron pruebas de comparaciones múltiples (intervalos de confianza simultáneos). Este análisis de varianza contempla una hipótesis de "no diferencia" en las medias de las poblaciones denominado hipótesis nula. Para determinar si esta hipótesis nula se cumple o no, se realizó una prueba "F" o "razón de varianza", en donde se confrontaron los valores del "F" calculados con el tabulado.

\section{RESULTADOS Y DISCUSIÓN}

Un resumen de los valores de resistencia ante esfuerzo de cizalle y de la adhesión, separados por tipo de tratamiento, tipo de adhesivo y tamaño de probeta se entregan en las tablas 1,2 y 3.

Los valores identificados como "Media" en las tablas 1, 2 y 3 corresponden al promedio de 20 probetas, tal como lo exige la norma. En la base de las tablas 1, 2 y 3 se encuentra una última serie de datos en la que se presenta el promedio de los cuatro adhesivos por tamaño de probeta.

Solo "Climatizado". En la tabla 1 se presenta el resumen de las medias de resistencia al cizalle y desgarro de fibras obtenidas luego de haber sometido las probetas solo a climatizado.

Tabla 1: Cizalle y adhesión probetas solo "Climatizadas"

\begin{tabular}{|c|c|c|c|c|c|}
\hline \multirow[b]{2}{*}{ Adhesivo } & \multirow{2}{*}{$\begin{array}{l}\text { Ancho de } \\
\text { probeta } \\
(\mathrm{mm})\end{array}$} & \multicolumn{2}{|c|}{ Resistencia a CIZALLE } & \multicolumn{2}{|c|}{ DESGARRO de fibras } \\
\hline & & $\begin{array}{l}\text { Media } \\
(\mathrm{MPa})\end{array}$ & $\begin{array}{c}\text { Desviación } \\
\text { estándar }\end{array}$ & $\begin{array}{l}\text { Media } \\
(\mathrm{MPa})\end{array}$ & $\begin{array}{c}\text { Desviación } \\
\text { estándar }\end{array}$ \\
\hline \multirow{3}{*}{$\mathbf{A}$} & 50,8 & 12,4 & 1,6 & 37 & 29 \\
\hline & 30,0 & 12,0 & 2,8 & 49 & 35 \\
\hline & 18,0 & 11,6 & 2,5 & 41 & 35 \\
\hline \multirow{3}{*}{ B } & 50,8 & 12,0 & 1,3 & 35 & 27 \\
\hline & 30,0 & 10,8 & 2,2 & 37 & 32 \\
\hline & 18,0 & 11,6 & 2,3 & 37 & 29 \\
\hline \multirow{3}{*}{ C } & 50,8 & 11,8 & 2,0 & 24 & 19 \\
\hline & 30,0 & 10,5 & 1,4 & 30 & 24 \\
\hline & 18,0 & 10,8 & 1,8 & 40 & 33 \\
\hline \multirow{3}{*}{ D } & 50,8 & 12,9 & 1,7 & 53 & 32 \\
\hline & 30,0 & 13,0 & 2,5 & 41 & 33 \\
\hline & 18,0 & 12,8 & 2,5 & 40 & 37 \\
\hline Promedio & 50,8 & 12,3 & 1,7 & 37 & 27 \\
\hline de $\operatorname{los} 4$ & 30,0 & 11,6 & 2,2 & 39 & 31 \\
\hline adhesivos & 18,0 & 11,7 & 2,3 & 39 & 33 \\
\hline
\end{tabular}


Para probetas solo climatizadas, el análisis estadístico, realizado con un nivel de confianza del 95\%, determinó que no hay diferencias significativas entre las resistencias al cizalle de los tres tipos de probeta.

Pese a lo anterior los resultados de la resistencia al cizalle permiten destacar que tres de los adhesivos (A, B y C) evidencian una disminución de la resistencia al reducir la superficie de cizalle de $19,35 \mathrm{~cm}^{2}$ a $11,43 \mathrm{~cm}^{2}$. El adhesivo D no muestra un cambio notorio en la resistencia. Al disminuir el tamaño de la probeta de $11,43 \mathrm{~cm}^{2}$ al mínimo ensayado, no se dan variaciones importantes en cizalle.

Con los resultados de la tabla 1 se preparó la figura 3, donde se presentan las variaciones de cizalle y adhesión bajo situación de "Climatizado" expresadas como porcentaje del valor obtenido con una probeta de sección 19,36 $\mathrm{cm}^{2}$ (ancho 50,8 mm), sin discriminar entre los adhesivos.

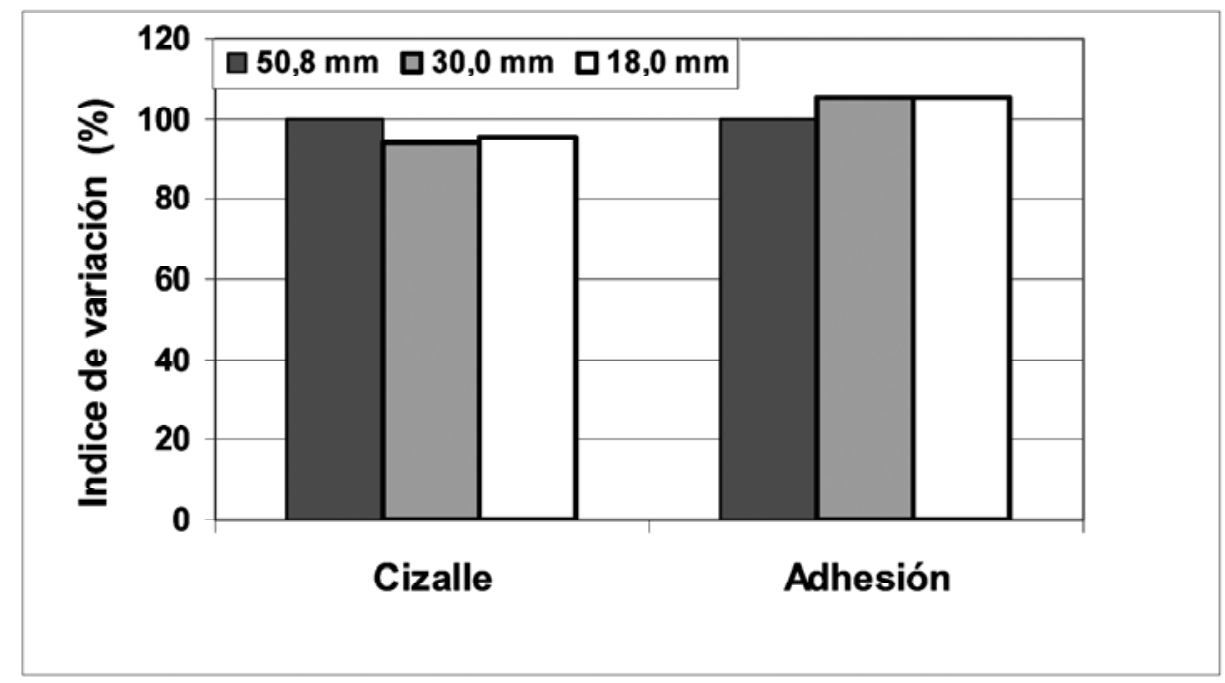

Figura 3: Solo "Climatizado": Variación de las propiedades cizalle y adhesión al cambiar la dimensión de la probeta.

En la figura 3 se observa una disminución de un $6 \%$ de la propiedad cizalle al pasar de 50,8 $\mathrm{mm}$ a $30,0 \mathrm{~mm}$. Posteriormente la resistencia permanece constante.

En el caso de la adhesión, el análisis estadístico del tratamiento Curado estableció la no existencia de diferencias significativas, al 95\% de nivel de confianza, entre los diferentes tipos de probeta.

Los adhesivos A, B y C muestran un comportamiento semejante y diferente del adhesivo D. Se observa una disminución de 5\% de la propiedad al disminuir la sección de cizalle de 19,35 cm² a 11,43 $\mathrm{cm}^{2}$. Con mayores disminuciones no hay cambios en la propiedad. Lo anterior se ve claramente expresado por los índices de variación presentados en la figura 3.

Tratamiento de Ebullición. Un resumen de las medias de resistencia al cizalle y desgarro de fibras obtenidas luego de haber tratado las probetas con agua a $100{ }^{\circ} \mathrm{C}$ se presenta en la tabla 2 .

Con un nivel de confianza del 95\%, las resistencias al cizalle de las probetas de 30,0 $\mathrm{mm}$ y $18,0 \mathrm{~mm}$ son estadísticamente diferentes. El análisis revela que no existen diferencias significativas entre las mencionadas con las de a las de 50,8 mm, no observándose una tendencia en los cambios de resistencia al variar el tamaño de la probeta para los cuatro adhesivos. 
Con los resultados de la tabla 2 se preparó la figura 4, donde se presentan las variaciones de cizalle y adhesión en el tratamiento de "Ebullición" expresadas como porcentaje del valor obtenido con una probeta de sección 19,35 $\mathrm{cm}^{2}$ (ancho 50,8 $\mathrm{mm}$ ), sin discriminar entre los adhesivos.

Tabla 2: Cizalle y Adhesión con el tratamiento "Ebullición"

\begin{tabular}{|c|c|c|c|c|c|}
\hline \multirow[b]{2}{*}{ Adhesivo } & \multirow{2}{*}{$\begin{array}{c}\text { Ancho de } \\
\text { probeta } \\
(\mathrm{mm})\end{array}$} & \multicolumn{2}{|c|}{ Resistencia a CIZALLE } & \multicolumn{2}{|c|}{ DESGARRO de fibras } \\
\hline & & $\begin{array}{l}\text { Media } \\
(\mathrm{MPa})\end{array}$ & $\begin{array}{c}\text { Desviación } \\
\text { estándar }\end{array}$ & $\begin{array}{l}\text { Media } \\
\text { (MPa) }\end{array}$ & $\begin{array}{l}\text { Desviación } \\
\text { estándar }\end{array}$ \\
\hline \multirow{3}{*}{$\mathbf{A}$} & 50,8 & 3,2 & 0,6 & 29 & 10 \\
\hline & 30,0 & 3,5 & 0,5 & 9 & 5 \\
\hline & 18,0 & 3,8 & 0,8 & 7 & 6 \\
\hline \multirow{3}{*}{ B } & 50,8 & 2,7 & 0.7 & 4 & 5 \\
\hline & 30,0 & 2,6 & 0,8 & 10 & 5 \\
\hline & 18,0 & 3,1 & 1,0 & 9 & 7 \\
\hline \multirow{3}{*}{ C } & 50,8 & 2,9 & 0,8 & 10 & 13 \\
\hline & 30,0 & 2,9 & 0,7 & 13 & 15 \\
\hline & 18,0 & 3,1 & 0,6 & 21 & 17 \\
\hline \multirow{3}{*}{ D } & 50,8 & 4,2 & 0,6 & 31 & 26 \\
\hline & 30,0 & 3,7 & 1,2 & 13 & 17 \\
\hline & 18,0 & 3,9 & 0,8 & 15 & 17 \\
\hline Promedio & 50,8 & 3,3 & 0,7 & 19 & 18 \\
\hline de $\operatorname{los} 4$ & 30,0 & 3,2 & 0,8 & 11 & 11 \\
\hline adhesivos & 18,0 & 3,5 & 0,8 & 13 & 12 \\
\hline
\end{tabular}

En la figura 4 es posible visualizar una disminución del 3\% de la propiedad cizalle al disminuir el tamaño de la probeta de 50,8 a 30,0 mm y un aumento del $6 \%$ de esta misma propiedad al determinarla con probetas de $18,0 \mathrm{~mm}$.

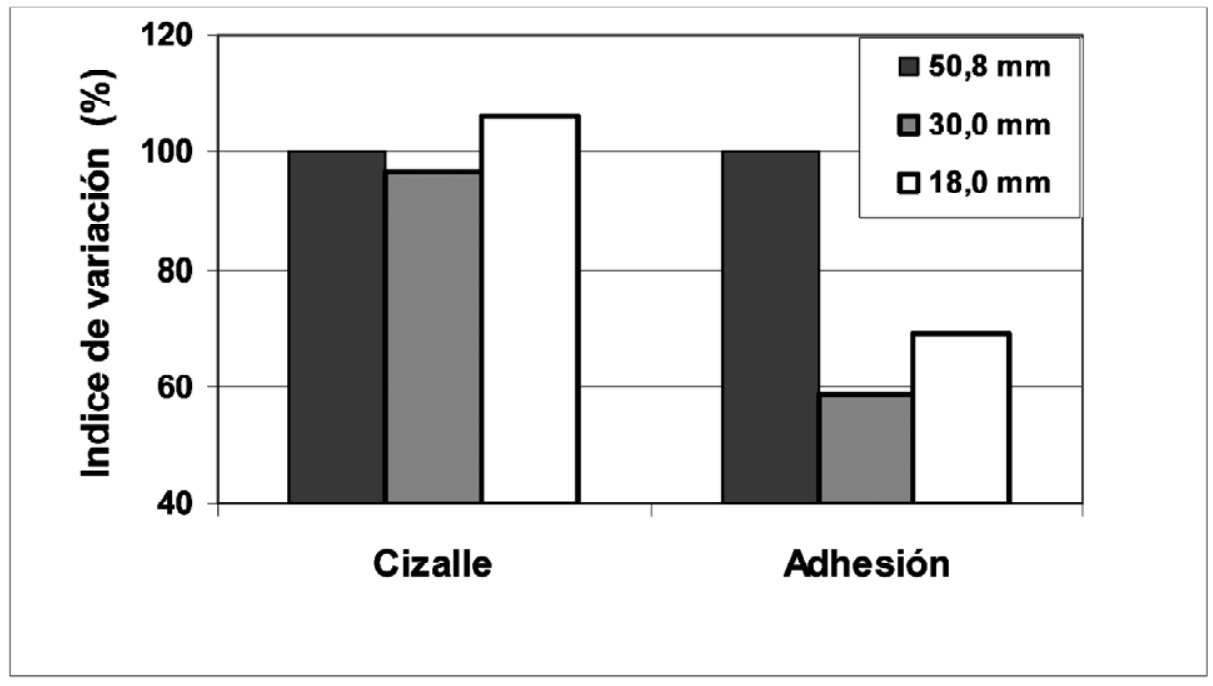

Figura 4: Con tratamiento de "Ebullición": Variación de las propiedades cizalle y adhesión al cambiar la dimensión de la probeta. 
En adhesión con un nivel de confianza del $95 \%$ en el tratamiento de ebullición, probetas de 30,0 y $18,0 \mathrm{~mm}$ son iguales y ambas son diferentes de $50,8 \mathrm{~mm}$.

En relación a los adhesivos, A tiende a aumentar su porcentaje de desgarro en la medida que aumenta el ancho de la probeta. C presenta un comportamiento contrario y los adhesivos B y D no presentan ninguna tendencia.

Tratamiento de "Vacío presión". En la tabla 3 se indican las medias de resistencia al cizalle y desgarro de fibras de las probetas posterior a ser sometidas a vacío y presión bajo agua.

Bajo este tratamiento el análisis estadístico al 95\% de confianza determinó diferencias significativas entre las resistencias al cizalle de las probetas de $50,8 \mathrm{~mm}$ y $30,0 \mathrm{~mm}$ con las de $18,0 \mathrm{~mm}$ de ancho.

Tabla 3: Cizalle y adhesión con el tratamiento "Vacío-Presión"

\begin{tabular}{|c|c|c|c|c|c|}
\hline \multirow[b]{2}{*}{ Adhesivo } & \multirow{2}{*}{$\begin{array}{c}\text { Ancho de } \\
\text { probeta } \\
(\mathrm{mm})\end{array}$} & \multicolumn{2}{|c|}{ Resistencia a CIZALLE } & \multicolumn{2}{|c|}{ DESGARRO de fibras } \\
\hline & & $\begin{array}{l}\text { Media } \\
\text { (MPa) }\end{array}$ & $\begin{array}{l}\text { Desviación } \\
\text { estándar }\end{array}$ & $\begin{array}{l}\text { Media } \\
\text { (MPa) }\end{array}$ & $\begin{array}{c}\text { Desviacion } \\
\text { estándar }\end{array}$ \\
\hline \multirow{3}{*}{$\mathbf{A}$} & 50,8 & 6,4 & 0,7 & 9 & 11 \\
\hline & 30,0 & 6,2 & 1,2 & 29 & 22 \\
\hline & 18,0 & 5,5 & 0,9 & 30 & 23 \\
\hline \multirow{3}{*}{ B } & 50,8 & 4,8 & 0,6 & 3 & 3 \\
\hline & 30,0 & 4,7 & 0,8 & 11 & 11 \\
\hline & 18,0 & 4,5 & 0,8 & 8 & 5 \\
\hline \multirow{3}{*}{$\mathbf{C}$} & 50,8 & 4,1 & 0.7 & 13 & 13 \\
\hline & 30,0 & 3,9 & 0,8 & 12 & 17 \\
\hline & 18,0 & 3,8 & 0,8 & 10 & 11 \\
\hline \multirow{3}{*}{ D } & 50,8 & 6,2 & 0,9 & 21 & 20 \\
\hline & 30,0 & 5,9 & 0,8 & 30 & 22 \\
\hline & 18,0 & 5,9 & 1,0 & 21 & 20 \\
\hline Promedio & 50,8 & 5,4 & 0,7 & 11 & 12 \\
\hline de $\operatorname{los} 4$ & 30,0 & 5,2 & 0,9 & 20 & 18 \\
\hline adhesivos & 18,0 & 4,9 & 0,9 & 17 & 15 \\
\hline
\end{tabular}

Todos los adhesivos mostraron una tendencia a disminuir la resistencia al cizalle al disminuir el tamaño del plano sometido a esfuerzo. El adhesivo D presentó igual resultado entre las probetas de 30,0 y $18,0 \mathrm{~mm}$

Con los resultados de la tabla 3 se preparó la figura 5, donde se presentan las variaciones de cizalle y adhesión en el tratamiento de "Vacío-Presión" expresadas como porcentaje del valor obtenido con probetas normalizadas (ancho 50,8 $\mathrm{mm}$ ), sin discriminar entre los adhesivos. 


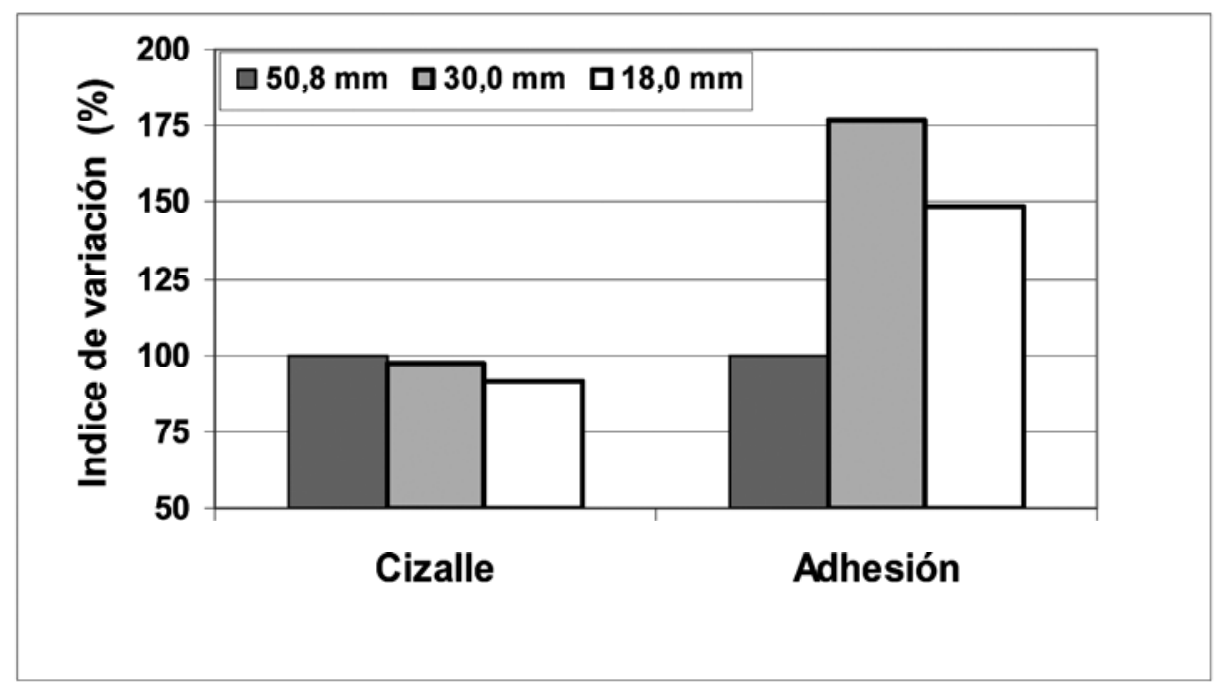

Figura 5: Con tratamiento "Vacío - Presión": Variación de las propiedades cizalle y adhesión al cambiar la dimensión de la probeta.

En la figura 5 se muestra que, para la propiedad cizalle, los valores obtenidos en probetas de 30,0 y $18,0 \mathrm{~mm}$ disminuyen aproximadamente en un 3 y $8 \%$ respectivamente al compararlos con el valor obtenido en probetas normalizadas.

En adhesión con un nivel de confianza del $95 \%$ en el tratamiento de Vacío-Presión, probetas de $30,0 \mathrm{~mm}$ y 18,0 son iguales y ambas son diferentes de 50,8

El adhesivo A tiende a aumentar su porcentaje de desgarro en la medida que disminuye el ancho de la probeta, $\mathrm{C}$ presenta tendencia contraria $\mathrm{y}$, sin discriminar por adhesivos, los porcentajes de desgarro de fibras son significativamente más altos en probetas de tamaño inferior al normalizado.

\section{CONCLUSIONES}

Al realizar ensayos de cizalle de uniones laminares con adhesivos tipo PVA según el procedimiento establecido en la norma ASTM D - 5751 se puede concluir que:

En situación de muestras climatizadas no existen diferencias significativas entre los diferentes tamaños de probeta, por lo tanto sería posible realizar este ensayo con probetas de ancho $\geq 18 \mathrm{~mm}$.

En probetas que serán sometidas a tratamientos de envejecimiento acelerado, debe respetarse el ancho normalizado $(50,8 \mathrm{~mm})$, tanto para determinar resistencia como para medir desgarro de fibras. 


\section{BIBLIOGRAFIA}

American Society for Testing and Materials. 1995. Standard Specification for Adhesives Used for Laminate Joints in Nonstructural Lumber. Designation D-5751.

Comité Europeo de Normalización. 1993. Clasificación de adhesivos no estructurales para uniones de madera y productos derivados de la madera. UNE EN 204. Asociación Española de Normalización y Certificación. Madrid, 7p.

Comité Europeo de Normalización. 1993. Clasificación de adhesivos no estructurales para uniones de madera y productos derivados de la madera. UNE EN 205. Asociación Española de Normalización y Certificación. Madrid, 13p.

Poblete, H.; Cuevas, H. 1998. Uniones endentadas y de canto con Pinus radiata. Experiencias sobre su calidad. Bosque 19(2): 77-84. 
